ARTICLE

\title{
Lanthanum(III) triggers AtrbohD- and jasmonic acid-dependent systemic endocytosis in plants
}

\author{
Mengzhu Cheng ${ }^{1,8}$, Lihong Wang ${ }^{1,2,8}$, Qing Zhou ${ }^{2}$, Daiyin Chao ${ }^{3}$, Shingo Nagawa ${ }^{4,5}$, Ding He ${ }^{2}$, Jiazhi Zhang ${ }^{2}$,
} Hui Li ${ }^{5,6}$, Li Tan ${ }^{5}$, Zhenhong Gu${ }^{5}$, Xiaohua Huang ${ }^{1 凶} \&$ Zhenbiao Yang $\mathbb{D}^{4,7 凶}$

Trivalent rare earth elements (REES) are widely used in agriculture. Aerially applied REEs enter leaf epidermal cells by endocytosis and act systemically to improve the growth of the whole plant. The mechanistic basis of their systemic activity is unclear. Here, we show that treatment of Arabidopsis leaves with trivalent lanthanum [ $\mathrm{La}(\mathrm{III})]$, a representative of REEs, triggers systemic endocytosis from leaves to roots. $\mathrm{La}(\mathrm{III})$-induced systemic endocytosis requires AtrbohD-mediated reactive oxygen species production and jasmonic acid. Systemic endocytosis impacts the accumulation of mineral elements and the development of roots consistent with the growth promoting effects induced by aerially applied REEs. These findings provide insights into the mechanistic basis of REE activity in plants.

\footnotetext{
${ }^{1}$ National and Local Joint Engineering Research Center of Biomedical Functional Materials, Jiangsu Collaborative Innovation Center of Biomedical Functional Materials, School of Chemistry and Materials Science, Nanjing Normal University, Nanjing, China. ${ }^{2}$ State Key Laboratory of Food Science and Technology, Jiangnan University, Wuxi, China. ${ }^{3}$ National Key Laboratory of Plant Molecular Genetics, Institute of Plant Physiology and Ecology, Shanghai Institutes for Biological Sciences, Chinese Academy of Sciences, Shanghai, China. ${ }^{4}$ Fujian Agriculture and Forestry University-University of California, Riverside, Joint Center for Horticultural Biology and Metabolomics, Haixia Institute of Science and Technology, Fujian Agriculture and Forestry University, Fuzhou, China. ${ }^{5}$ Shanghai Center for Plant Stress Biology, Shanghai Institute of Biological Sciences, Chinese Academy of Sciences, Shanghai, China. ${ }^{6}$ School of Life Sciences, East China Normal University, Shanghai, China. ${ }^{7}$ Department of Botany and Plant Sciences, Center for Plant Cell Biology, Institute of Integrative Genome Biology, University of California, Riverside, CA, USA. ${ }^{8}$ These authors contributed equally: Mengzhu Cheng, Lihong Wang. ${ }^{凶}$ email: huangxiaohuanjnu@yahoo. com; yang@ucr.edu
} 
T rivalent rare earth elements (REEs) have long been known to promote plant growth and are widely used as growth regulators to improve crop productivity mainly via foliar application ${ }^{1-3}$. Interestingly, foliar application of REEs induces systemic impacts on plant growth and development (such as changes in leaf area and weight, root length and weight) and physiological activities (such as changes in photosynthesis in leaves and mineral element levels in roots), which are commonly observed 7 days and $24 \mathrm{~h}$ after the application of REEs, respectively ${ }^{2,4,5}$. In addition, REEs are widely used materials in industry, medicine, military, etc. ${ }^{6-8}$. Due to the ever-increasing usage, REEs increasingly accumulate in the environment and living organisms ${ }^{9}$, raising significant concerns for human health $^{9-12}$. Thus, REEs have become important elements that affect living organisms ${ }^{9-12}$. However, the modes of REE activity in living organisms have been puzzling researchers over the past 100 years. In particular nothing is known about the cellular and molecular mechanisms by which locally applied REEs trigger a systemic response in plants $4,13-15$. Recently, our studies using interdisciplinary approaches, such as electron microscopy autoradiography of REE radioisotopes, including lanthanum $\left[{ }^{140} \mathrm{La}\right.$ (III)], cerium $\left[{ }^{141} \mathrm{Ce}(\mathrm{III})\right]$, and terbium $\left[{ }^{160} \mathrm{~Tb}(\mathrm{III})\right]$, and total internal reflection fluorescence microscopy, have demonstrated that aerially applied REEs for $12 \mathrm{~h}$ initiate their own endocytosis in plant leaf cells ${ }^{4,14,15}$. Such endocytosis has not been observed in plants treated with other metal elements ${ }^{4}$. The unique effects of REEs have drawn a great deal of attention from scientists in various fields ${ }^{16-23}$. However, the mechanisms for the systemic responses in plants triggered by aerial REE application remain unclear.

In this study, we find that $\mathrm{La}(\mathrm{III})$ application on Arabidopsis leaves activates the production of systemic signal(s) inducing endocytosis in roots and that this systemic response requires coordinate action of AtrbohD (an NADPH oxidase) with jasmonic acid (JA). Systemic endocytosis changes the accumulation of mineral elements in roots and the growth of the whole plant, including leaf expansion, primary root growth, and lateral root formation. These findings have demonstrated the existence of systemic endocytosis, suggested a mechanistic basis for systemic signaling mediated by the coordinate action of AtrbohD and JA, and created opportunities for the study of systemic signaling and the mode of REE activity in living organisms.

\section{Results}

$\mathrm{La}$ (III) induces systemic endocytosis. We used several complementary methods to visualize endocytosis induced by aerially applied 30 or $80 \mu \mathrm{M} \mathrm{LaCl}_{3}$ that has a respective promotive or inhibitory effect on the growth and physiological activities of the whole Arabidopsis plant (Supplementary Table 1). First, we confirmed $\mathrm{LaCl}_{3}$-induced endocytosis in leaf epidermal cells stained with $\mathrm{N}$-(3-triethylammoniumpropyl)-4-(4-diethylaminophenylhexatrienyl) (FM4-64), a fluorescent dye that only penetrates cell membranes via endocytosis ${ }^{24,25}$, as previously reported $^{4,15}$. Treatment of wild-type Arabidopsis (Col-0) leaves with $30 \mu \mathrm{M} \mathrm{LaCl}{ }_{3}$ greatly increased the frequency of endocytosis (the percentage of cells exhibiting endocytosis) and the number of endocytic vesicles per cell (the average number of endocytic vesicles, i.e., dot- or circle-like structures stained with FM4-64) in leaf epidermal cells (Supplementary Fig. 1a, b), respectively. Next, transmission electronic microscopy (TEM) was used to observe the sizes of $30 \mu \mathrm{M} \mathrm{LaCl}_{3}$-induced vesicles and their likely biogenesis because TEM can determine the morphology of endocytic vesicles with high resolution ${ }^{26}$. The TEM images showed that these vesicles varied in sizes mainly around $100-200 \mathrm{~nm}$ in diameters localized to the vicinity of the plasma membrane (PM) and appeared to result from the PM invagination (Supplementary Fig. 1c, d). Treatment with $80 \mu \mathrm{M} \mathrm{LaCl}$ induced more pronounced changes in vesicle formation (Supplementary Fig. 1). In mock-treated epidermal cells, we did not observe such vesicles and the PM invagination (Supplementary Fig. 1). These results are consistent with our previous results observed via electron microscopy autoradiography of ${ }^{140} \mathrm{La}(\mathrm{III})$ radioisotopes ${ }^{4,15}$.

Because the majority of endocytic events reported in plant cells are clathrin-mediated endocytosis $(\mathrm{CME})^{24,27}$, we tested whether $\mathrm{LaCl}_{3}$-induced endocytosis in leaf epidermal cells was also clathrin-mediated using a line expressing green fluorescent protein (GFP)-tagged clathrin light chain 1 (CLC1, an essential subunit of clathrin coats ${ }^{28}$ ) and by co-staining with FM4-64. We found that CLC1-GFP-labeled vesicles were only observed in the epidermal cells of leaves treated with 30 or $80 \mu \mathrm{M} \mathrm{LaCl}$ (Supplementary Fig. 2a). CLC1-GFP showed overlapping distribution with FM4-64 both on the PM and the membrane of $\mathrm{LaCl}_{3}$-induced vesicles (Supplementary Fig. 2a). These results suggest that $\mathrm{LaCl}_{3}$ treatment induced CME in leaf epidermal cells. The frequency of CME and the number of CLC1-GFP-labeled vesicles per cell in leaves treated with $80 \mu \mathrm{M} \mathrm{LaCl}_{3}$ were much higher than in those treated with $30 \mu \mathrm{M} \mathrm{LaCl}{ }_{3}$ (Supplementary Fig. 2b). Importantly, time-lapse imaging showed that $\mathrm{LaCl}_{3}$ treatment induced the budding of CLC1-GFP-labeled membrane and their pinching off from the PM in leaf epidermal cells (Supplementary Movie 1). Taken together, our results demonstrate that $\mathrm{LaCl}_{3}$ treatment on Arabidopsis leaves induces CME in leaf epidermal cells. This conclusion is further supported by inhibiting CME using genetic approaches (see below). It is noteworthy that some FM4-64- or CLC1-GFP-labeled vesicles $>200 \mathrm{~nm}$ were observed in confocal laser scanning microscope (CLSM) images (Supplementary Figs. 1a and 2a), which may result from the fusion of vesicles (Supplementary Movie 1) and/or lower spatial resolution of CLSM ${ }^{26}$.

It was reported that $\mathrm{LaCl}_{3}$-triggered endocytosis in plant leaves enhanced the contents of nutrient elements in leaf cells ${ }^{4}$. The nutrient elements of plants mainly come from their absorption from the soil by roots. Furthermore, endocytosis may contribute to the uptake of nutrient elements ${ }^{29,30}$. Thus, we speculated that the increased contents of nutrient elements in leaf cells could result from the increased endocytosis in root cells. We observed endocytosis in root epidermal cells $12 \mathrm{~h}$ after treatment of Col-0 leaves with $\mathrm{LaCl}_{3}$. Indeed, this treatment greatly enhanced endocytosis in root epidermal cells (Fig. 1a, b). Compared with the mock-treated control plants, the number of FM4-64-stained vesicles per root epidermal cell in $30 \mu \mathrm{M} \mathrm{LaCl}_{3}$-treated plants increased by $45 \%$ (Fig. 1a, b). Meanwhile, the frequency of root epidermal cells containing FM4-64-stained vesicles was 77\% in $30 \mu \mathrm{M} \mathrm{LaCl}_{3}$-treated plants, compared to $54 \%$ of root epidermal cells in mock-treated control plants (Fig. 1a, b). Treatment of leaves with $80 \mu \mathrm{M} \mathrm{LaCl}_{3}$ induced a much greater increase in the frequency of cells with FM4-64-stained vesicles and the number of these vesicles per cell in the root epidermis (Fig. 1a, b). Moreover, CLC1-GFP exhibited overlapping distribution with FM4-64 both on the PM and vesicles in root epidermal cells (Fig. 1c-e). To assess whether the $\mathrm{LaCl}_{3}$-induced increase in FM4-64- and CLC1-GFP-co-labeled vesicles resulted from the enhanced CME in leaf epidermal cells or the inhibition of endosomal recycling in root epidermal cells, we next constructed the grafted plants in which shoots from Col-0 were grafted onto roots from the ap2 $\mu$-1 mutant [a loss-of-function mutation in adaptor protein 2 (AP2), the CME-specific clathrin adapter ${ }^{15,29}$. In these grafted plants whose leaves were treated with $\mathrm{LaCl}_{3}$, the number of FM4-64-stained vesicles in root epidermal cells was lower (Fig. 1f, g), compared with $\mathrm{LaCl}_{3}$-treated Col- 0 plants or grafted control plants, in which Col-0 shoots were grafted onto 

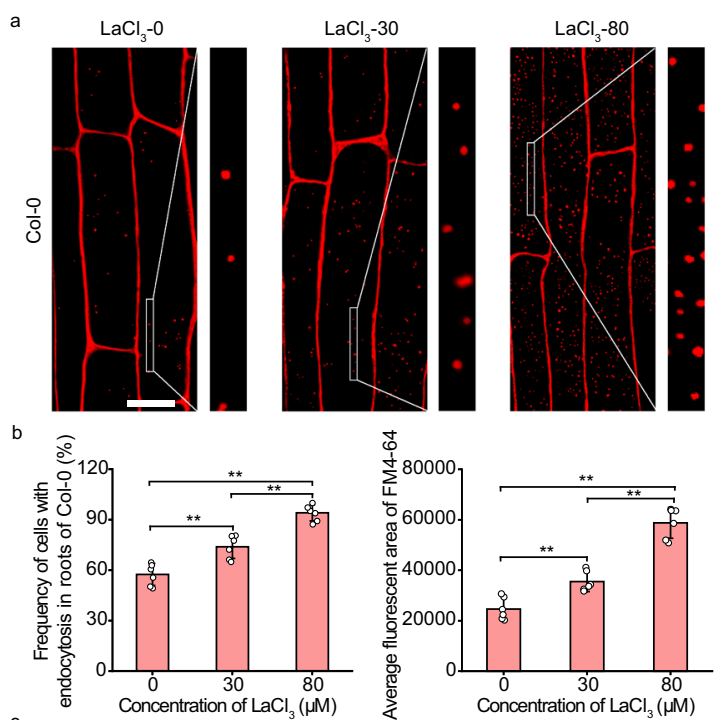

फे
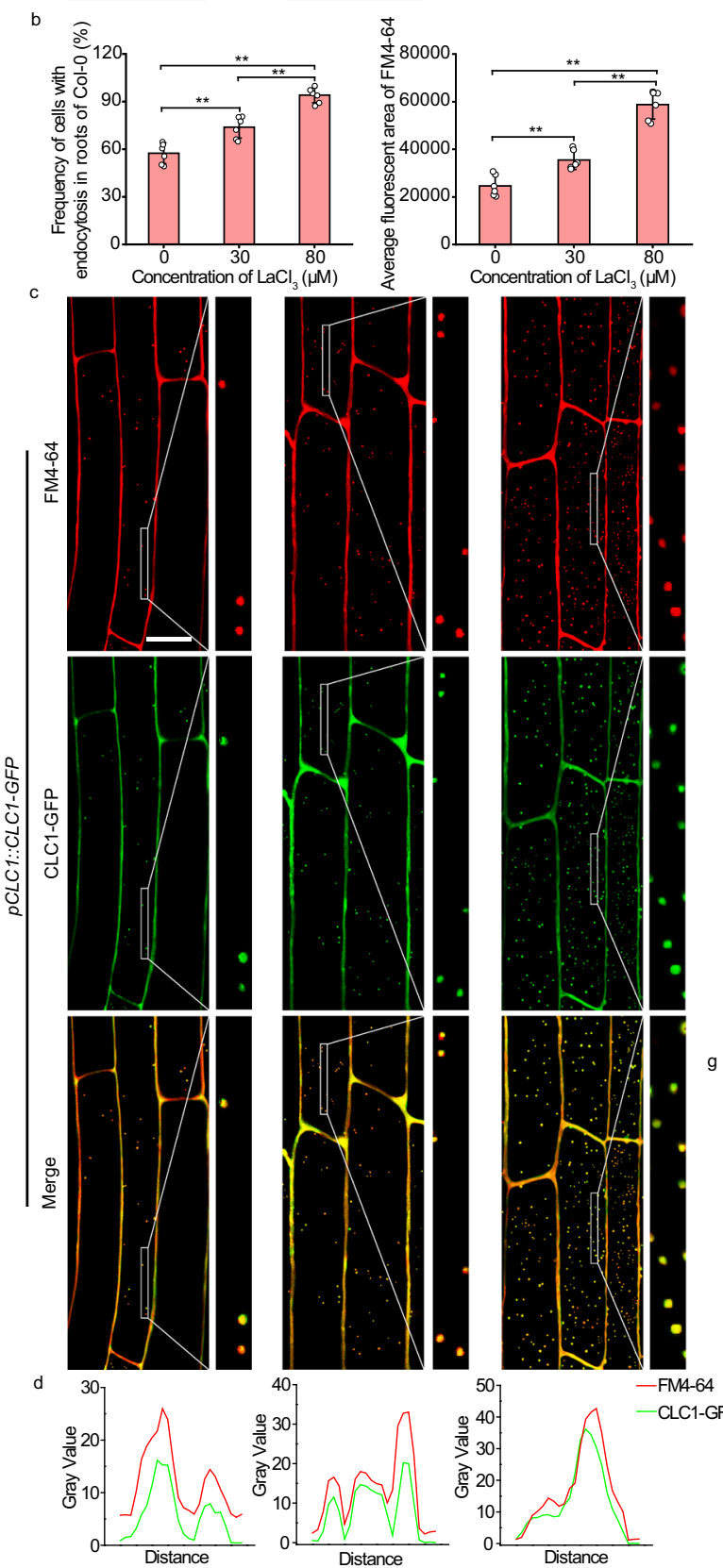

Col-0 roots (Fig. 1f, g). These results suggest that $\mathrm{LaCl}_{3}$ treatment of leaves not only induces local CME in leaf epidermal cells but also systemically promotes CME in root epidermal cells.

Our previous study showed that $\mathrm{LaCl}_{3}$-induced endocytosis in leaf epidermis cells is not due to $\mathrm{Cl}^{-}$or salt stress ${ }^{4}$. We next asked whether $\mathrm{LaCl}_{3}$-induced systemic endocytosis resulted from $\mathrm{Cl}^{-}$or osmotic stress. To this end, we observed endocytosis in $\mathrm{NaCl}$-treated
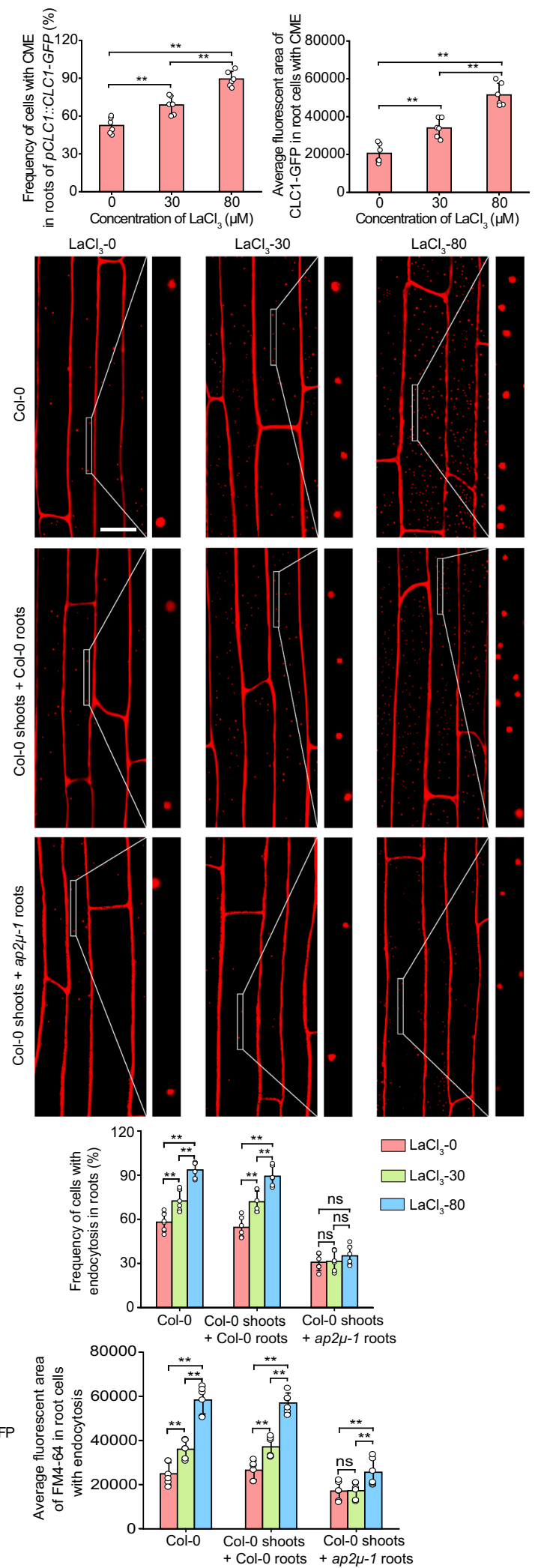

Col-0 plants, in which the molar concentration of $\mathrm{Cl}^{-}$in $\mathrm{NaCl}$ equaled to that in $\mathrm{LaCl}_{3}$. Twelve hours after treatment of leaves with $\mathrm{NaCl}$, endocytosis in leaf and root epidermal cells was not changed, compared to the control cells (Supplementary Fig. 3). It was reported that salt stress often affects the growth and development of plant cells by changing osmotic pressure ${ }^{31}$, and osmotic stress can modulate the balance between exocytosis and $\mathrm{CME}^{32}$. To assess whether $\mathrm{LaCl}_{3}$ 
Fig. $1 \mathrm{LaCl}_{\mathbf{3}}$ induced CME in root cells. a The representative CLSM images of Col-0 root cells. Leaves of Col-0 were treated with 0,30 , or $80 \mu \mathrm{M} \mathrm{LaCl}{ }_{3}$ for $12 \mathrm{~h}$ and then roots were stained with FM4-64. Bar $=10 \mu \mathrm{m}$. $\mathbf{b}$ Quantitative analysis of the frequency of root cells with endocytosis and average fluorescent area of FM4-64 in Col-0 root cells (data come from a). c The representative CLSM images of $p$ CLC1::CLC1-GFP root cells. Leaves of $p$ CLC1::CLC1-GFP were treated with 0,30 , or $80 \mu \mathrm{M} \mathrm{LaCl}_{3}$ for $12 \mathrm{~h}$ and then roots were stained with $\mathrm{FM} 4-64$. Bar $=10 \mu \mathrm{m}$. $\mathbf{d}$ The intensity plot of corresponding solid boxes shown in (c). e Quantitative analysis of the frequency of $p C L C 1:: C L C 1-G F P$ root cells with CME and average fluorescent area of CLC1-GFP in these cells (data come from $\mathbf{c}$ ). f The representative CLSM images of Col-0, and the grafted Col- 0 and ap $2 \mu-1$ (shoots from Col-0 were grafted onto Col-0 and ap $2 \mu-1$ roots) root cells. Leaves of all of these Arabidopsis were treated with 0,30 , or $80 \mu \mathrm{M} \mathrm{LaCl} 3 \mathrm{for} 12 \mathrm{~h}$ and then roots were stained with $\mathrm{FM} 4-64$. Bar $=10 \mu \mathrm{m}$. $\mathbf{g}$ Quantitative analysis of the frequency of root cells with endocytosis and average fluorescent area of FM4-64 in these cells of Col-0, and the grafted Col-0 and ap $2 \mu-1$ (shoots from Col-0 were grafted onto Col-0 and ap2 $\mu-1$ roots) root cells (data come from $\mathbf{f}$ ). In $\mathbf{a}$, c, and $\mathbf{f}$, representative images from six independent measurements and three biological replicates (each replicate represents an independently treated plant) per measurement are represented, and the right sides of the CLSM images are the enlarged areas of solid boxes. In (b, e, g), values shown are means \pm SEM, one-way ANOVA analysis with LSD multiple comparisons test $\left(n=6,{ }^{\star \star} p<0.01\right.$, n.s.: no significance).

treatment caused osmotic stress in leaf epidermal cells, we measured osmotic pressure in Col- 0 leaves and roots $12 \mathrm{~h}$ after $\mathrm{LaCl}_{3}$ treatment. Treatment of leaves with 30 or $80 \mu \mathrm{M} \mathrm{LaCl}_{3}$ for $12 \mathrm{~h}$ did not cause changes in osmotic pressure in either leaves or roots (Supplementary Fig. 4). Therefore, $\mathrm{LaCl}_{3}$-induced systemic endocytosis specifically results from $\mathrm{La}(\mathrm{III})$, but is not associated with $\mathrm{Cl}^{-}$or osmotic stress.

Systemic endocytosis induced by $\mathrm{La}(\mathrm{III})$ is independent of $\mathrm{La}$ (III) in roots. To assess whether the induction of systemic endocytosis could result from possible transport of $\mathrm{La}$ (III) from leaves to roots, we determined the levels of $\mathrm{La}(\mathrm{III})$ in leaves and roots $12 \mathrm{~h}$ after treatment of leaves with $\mathrm{La}$ (III) using inductively coupled plasma mass spectrometry (ICP-MS). We found the accumulation of $\mathrm{La}$ (III) in leaves (Supplementary Fig. 5), but did not detect $\mathrm{La}$ (III) in roots (Supplementary Fig. 5). This result suggests that $12 \mathrm{~h}$ after treatment of leaves with $\mathrm{La}$ (III), $\mathrm{La}$ (III) was not transported to roots, which is in agreement with our previous report that very little REEs move from leaves to roots $48 \mathrm{~h}$ after treatment of leaves with REEs ${ }^{33}$. Therefore, systemic endocytosis induced by $\mathrm{La}$ (III) is independent of $\mathrm{La}(\mathrm{III})$ in roots.

La(III)-induced endocytosis in leaf cells triggers a systemic endocytosis signal. The $\mathrm{La}(\mathrm{III})$-induced systemic endocytosis could result from one or more of the following possible mechanisms: (1) aerially applied La(III) directly induces a systemic signal translocated from leaves to roots, (2) La(III)-induced endocytosis in leaf cells or locally internalized $\mathrm{La}$ (III) generates a systemic signal transported to roots. To distinguish these possible mechanisms, we used several methods to block La(III)-induced endocytosis in leaves. We first performed grafting experiments to investigate whether blocking $\mathrm{La}(\mathrm{III})$-induced endocytosis in leaves by the chc1-3 mutation [a loss-of-function mutation in clathrin heavy chain $1(\mathrm{CHC} 1)^{34}$ ] affects this systemic endocytosis. In these experiments, shoots from the chc1-3 mutant were grafted onto roots from Col-0 (Fig. $2 \mathrm{a}-\mathrm{d}$ ). In these grafted plants, $\mathrm{La}$ (III)-induced endocytosis in both leaf and root epidermal cells was greatly reduced (Fig. $2 \mathrm{a}-\mathrm{d}$ ). We then conducted another grafting experiment, in which shoots from dexamethasone (DEX)-inducible Venus-CANTH line were grafted onto roots from Col-0. CANTH is the C-terminal region of the ANTHcontaining CME adaptor protein, and is shown to specifically block CME when it is fused with Venus and expressed under DEX-inducible system ${ }^{15,35}$. As expected, DEX-induced expression of Venus-CANTH in leaves (Supplementary Fig. 6) blocked $\mathrm{La}$ (III)-induced endocytosis in leaf epidermal cells (Fig. 2a, b). Importantly, epidermal cells of the grafted Col-0 roots exhibited a reduction in $\mathrm{La}(\mathrm{III})$-induced systemic endocytosis (Fig. 2c, d) when the expression of Venus-CANTH was specifically induced in the leaves of grafted plants, compared to the mock-treated control Col-0 and grafted control plants (Col-0 shoots were grafted onto Col-0 roots). Furthermore, treatment of Col-0 leaves with tyrphostin A23 (TyrA23), an inhibitor of CME that functions through inducing cytoplasmic acidification ${ }^{36}$, also drastically decreased $\mathrm{La}$ (III)-enhanced systemic endocytosis in Col-0 root cells (Supplementary Fig. 7). Taken together, these results suggest that $\mathrm{La}$ (III)-induced endocytosis or $\mathrm{La}$ (III) internalized by endocytosis in leaves leads to the production of a systemic signal to induce endocytosis in roots.

We next assessed whether this systemic endocytosis is induced by the internalized $\mathrm{La}$ (III) or $\mathrm{La}$ (III)-induced endocytosis in leaves. We observed endocytosis in flagellin 22 (flg22)-treated Col- 0 because the stimulation with flg22 induced its own internalization via $\mathrm{CME}^{37}$. In Col-0 plants whose leaves were treated with flg22, systemic endocytosis was also induced (Supplementary Fig. 8). Therefore, we conclude that La(III)induced endocytosis in leaves induced systemic endocytosis in roots in Arabidopsis.

La(III)-induced systemic endocytosis requires AtrbohD. A previous study showed the presence of NADPH oxidase in endocytic vesicles ${ }^{38}$. Furthermore, NADPH oxidase-dependent reactive oxygen species (ROS) have been implicated as systemic signals in plants ${ }^{39-44}$. Therefore, we speculated that NADPH oxidase-mediated ROS might play important roles in transmitting endocytic signals from leaves to roots. To test this hypothesis, we first sprayed diphenyliodonium (DPI), an inhibitor of NADPH oxidase $^{45}$, on the surface of Col-0 leaves and observed endocytosis in root epidermal cells $12 \mathrm{~h}$ after $\mathrm{La}(\mathrm{III})$ treatment on Col-0 leaves. The DPI treatment greatly reduced $\mathrm{La}(\mathrm{III})$-enhanced endocytosis in root epidermal cells (Fig. 3a, b). Moreover, the application of DPI on stems also greatly reduced endocytosis in root epidermal cells from the same plant whose leaves were treated with $\mathrm{La}$ (III) (Fig. 3a, b). These results support the hypothesis that active NADPH oxidase is required for the production of the signal transmitted from leaves to roots to induce systemic endocytosis.

In Arabidopsis, AtrbohD and AtrbohF are two key NADPH oxidases responsible for the production of ROS second messengers induced by various primary signals ${ }^{39,41,46}$. To further determine the function of NADPH oxidase in transmitting systemic endocytosis, we first tested the expression levels of AtrbohD and AtrbohF transcripts in leaves and roots $12 \mathrm{~h}$ after treatment of leaves with $\mathrm{La}(\mathrm{III})$. We found that in both leaves and roots, the level of AtrbohD transcript, but not AtrbohF transcript, was greatly increased by treatment of leaves with $\mathrm{La}$ (III) (Fig. 3c, d). Importantly, La(III)-induced systemic endocytosis in root epidermal cells of atrbohD mutant ${ }^{46}$ was greatly reduced compared to Col-0 when leaves were treated with La(III) for $12 \mathrm{~h}$ (Fig. 3a, b). These results suggest that AtrbohD plays a critical role in $\mathrm{La}(\mathrm{III})$-induced systemic endocytosis. 

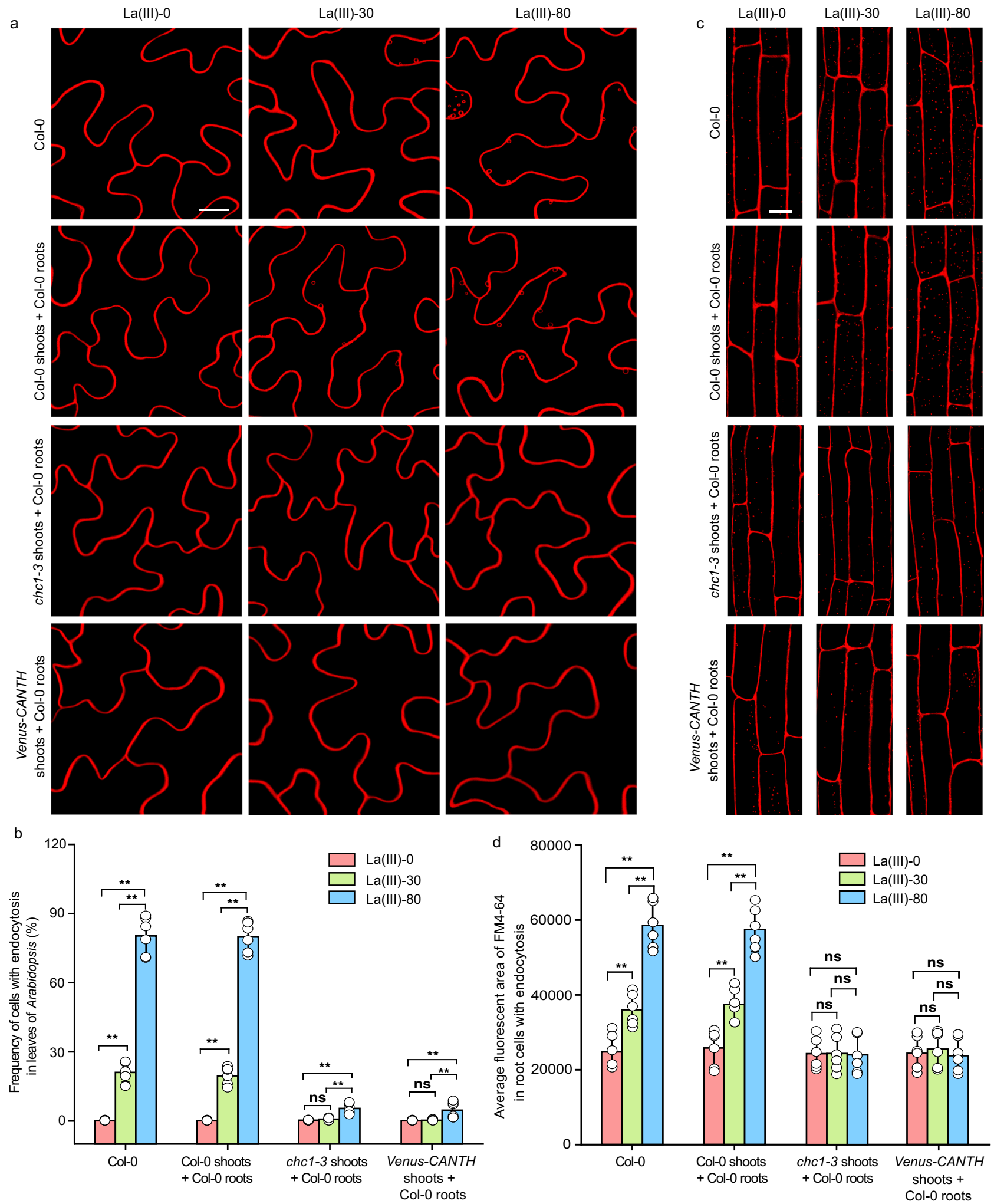

We then tested whether AtrbohD plays a role in generating and/or transmitting the systemic endocytosis signal using reciprocal grafting experiments. The atrbohD shoots were grafted onto Col-0 roots, and Col-0 shoots were grafted onto atrbohD roots, and their leaves were treated with $\mathrm{La}(\mathrm{III})$. In both types of grafted plants, the systemic endocytosis in root epidermal cells was greatly compromised compared to Col-0 (Fig. 3a, b). Thus, AtrbohD is required for triggering systemic endocytosis in roots

following the production of a systemic signal from leaves. Taken together, our results suggest that AtrbohD plays a key role in generating and/or transmitting a $\mathrm{La}$ (III)-induced long-distance signal from leaves to induce endocytosis in root cells.

AtrbohD transmits La(III)-induced long-distance endocytic signal through JA. How does AtrbohD transmit the endocytic 
Fig. 2 La(III)-induced endocytosis or La(III) internalized by endocytosis in leaves induced systemic endocytosis. a The representative CLSM images of Col-0, and the grafted Col-0, chc1-3, and DEX-inducible Venus-CANTH (shoots from Col-0, chc1-3, or DEX-inducible Venus-CANTH were grafted onto Col-0 roots) leaf cells treated with 0,30 , or $80 \mu \mathrm{M} \mathrm{La}$ (III) for $12 \mathrm{~h}$ and stained with FM4-64. Bar $=5 \mu \mathrm{m}$. b Quantitative analysis of leaf cells with endocytosis in Col-0, the grafted Col-0, chc1-3, and DEX-inducible Venus-CANTH (shoots from Col-0, chc1-3 or DEX-inducible Venus-CANTH were grafted onto Col-0 roots) (data come from a). c The representative CLSM images of Col-0, and the grafted Col-0, chc1-3, and DEX-inducible Venus-CANTH (shoots from Col0, chc1-3, or DEX-inducible Venus-CANTH were grafted onto Col-0 roots). The four kinds of Arabidopsis leaves were treated with 0, 30, or $80 \mu \mathrm{M}$ La(III) for $12 \mathrm{~h}$ and then roots were stained with FM4-64. Bar $=10 \mu \mathrm{m}$. d Quantitative analysis of the average fluorescent area of FM4-64 in Col-0, and the grafted Col-0, chc1-3, and DEX-inducible Venus-CANTH (shoots from Col-0, chc1-3, or DEX-inducible Venus-CANTH were grafted onto Col-0 roots) root cells with endocytosis (data come from $\mathbf{c}$ ). In ( $\mathbf{a}, \mathbf{c}$ ), representative images from six independent measurements and three biological replicates (each replicate represents an independently treated plant) per measurement are represented. In (b, d), values shown are means \pm SEM, one-way ANOVA analysis with LSD multiple comparisons test $\left(n=6,{ }^{\star \star} p<0.01\right.$, n.s.: no significance).

signal from leaves to roots after $\mathrm{La}(\mathrm{III})$ induces endocytosis in leaves? It has been proposed that NADPH oxidase-dependent ROS production mediates long-distance signaling triggered by diverse stimuli ${ }^{39-44}$, and the volatile plant hormone JA has also been implicated in long-distance signaling induced by biotic and abiotic stresses ${ }^{41,47-49}$. To assess whether JA participates in La (III)-triggered long-distance signal transmission to regulate endocytosis in roots, we first determined the levels of JA in leaves and roots $12 \mathrm{~h}$ after $\mathrm{La}(\mathrm{III})$ treatment on Col-0 leaves using liquid chromatography-MS (LC-MS). $\mathrm{La}(\mathrm{III})$ treatment greatly increased the levels of JA in Col-0 leaves and roots, suggesting a link of JA to $\mathrm{La}(\mathrm{III})$-triggered long-distance signal transmission (Supplementary Table 2). The atrbohD mutation compromised the increase in the levels of JA in leaves and roots in response to $\mathrm{La}(\mathrm{III})$ treatment (Supplementary Table 2). This result suggests a critical role for AtrbohD in the regulation of $\mathrm{La}(\mathrm{III})$-induced JA accumulation and a potential connection between JA and AtrbohD in $\mathrm{La}(\mathrm{III})$-triggered systemic signaling.

To test the role of endogenous JA in La(III)-triggered systemic signaling, we used a 12-oxophytodienoate reductase 3 mutant (opr3), which is impaired in JA synthesis and is defective in its long-distance signal transduction induced by biotic and abiotic stress $^{48,49}$. The opr 3 mutation greatly compromised systemic endocytosis in root epidermal cells induced by $\mathrm{La}(\mathrm{III})$ treatment on leaves (Fig. 4b, e), compared with those in Col-0 root epidermal cells (Fig. 4a, e). Application of JA on opr3 leaves rescued these defects in $\mathrm{La}(\mathrm{III})$-induced systemic responses (Fig. 4c, e). Furthermore, OPR3 transcript levels in roots were greatly increased $12 \mathrm{~h}$ after treatment of Col-0 leaves with $\mathrm{La}(\mathrm{III})$ (Supplementary Fig. 9). Because CORONATINE-INSENSITIVE 1 (COI1) is required for JA signaling ${ }^{47}$, we next asked whether COI1-dependent JA signaling participates in the regulation of La (III)-induced systemic responses. We sprayed $\mathrm{La}$ (III) on the surface of coil (impaired in JA signaling ${ }^{50}$ ) leaves and observed changes in endocytosis in root epidermis cells. In coil, the effects of $\mathrm{La}(\mathrm{III})$ on the systemic endocytosis were also greatly compromised compared to Col-0 (Fig. 4d, e). Taken together, these results indicate that $\mathrm{La}(\mathrm{III})$-induced JA biosynthesis and COI1-dependent JA signaling cooperate with AtrbohD in La(III)triggered systemic signaling to regulate endocytosis.

It was reported that treatment with a low concentration $(5 \mu \mathrm{M})$ of methyl jasmonate (MeJA) inhibited PIN2 endocytosis in COI1dependent manner, while a higher concentration $(50 \mu \mathrm{M})$ reduced PIN2 distribution to the $\mathrm{PM}^{51}$. Because $\mathrm{La}(\mathrm{III})$ induced JA accumulation in both leaves and roots only to sub-micromolar levels, we next asked whether sub-micromolar JA can activate endocytosis in roots. Treatment of roots with $50 \mathrm{nM} \mathrm{JA}$ for $12 \mathrm{~h}$ activated endocytosis in root epidermal cells (Supplementary Fig. 10). This is in contrast to the reported inhibition of PIN2 endocytosis by treatment with $5 \mu \mathrm{M}$ MeJA for $16 \mathrm{~h}^{51}$. This result suggests that our observed role of JA in the systemic regulation of endocytosis differs from the inhibition of PIN2 distribution to the
PM by high concentrations of MeJA. Thus, the La(III)-triggered systemic regulation of endocytosis by the cooperative action of AtrbohD and JA is unique. How AtrbohD coordinates with JA in the systemic signaling is worthy of future investigation.

La(III)-induced systemic endocytosis regulates the accumulation of mineral elements in roots and the growth of the whole plant. We next asked whether the systemic endocytosis induced by $\mathrm{La}(\mathrm{III})$ is involved in the regulation of $\mathrm{La}(\mathrm{III})$-induced systemic responses of plant growth. To address this question, we first measured the levels of mineral nutrient elements in the roots of Col-0 plants, whose leaves were treated with $\mathrm{La}(\mathrm{III})$. ICP-MS analysis showed that $24 \mathrm{~h}$ after treatment of leaves with $\mathrm{La}(\mathrm{III})$, the levels of major mineral nutrient elements (such as $\mathrm{K}, \mathrm{Ca}, \mathrm{Mg}$, $\mathrm{P}, \mathrm{Fe}$, and $\mathrm{Zn}$ ) in roots were greatly increased (Supplementary Table 3). Interestingly, treatment with TyrA23 or DPI on leaves negated the accumulation of these mineral nutrient elements induced by aerially applied $\mathrm{La}(\mathrm{III})$ (Supplementary Table 3 ). These data suggest that $\mathrm{La}(\mathrm{III})$-induced systemic endocytosis increased the accumulation of mineral nutrient elements in roots.

To assess whether the $\mathrm{La}(\mathrm{III})$-induced systemic endocytosis may play a role in the regulation of plant growth and development, we measured total leaf areas, primary root length, and lateral root numbers in Col-0 plants, whose leaves were treated with $\mathrm{La}(\mathrm{III})$ or co-treated with $\mathrm{La}(\mathrm{III})$ and $\mathrm{TyrA23}$, or $\mathrm{La}$ (III) and DPI. The results showed that the La(III)-induced effects on leaf expansion, primary root growth, and lateral root formation were all suppressed by co-treatment with either TyrA23 or DPI (Supplementary Table 4). Therefore, aerially applied REEs affect the growth of the whole plant apparently through their regulation of systemic endocytosis.

\section{Discussion}

In this study, we have provided convincing evidence that $\mathrm{La}(\mathrm{III})-$ activated endocytosis in Arabidopsis leaves generates an NADPH oxidase-dependent systemic signal to induce endocytosis in roots (Figs. 1-5, Supplementary Figs. 1-10, and Supplementary Tables 1 and 2). This finding begs for many interesting questions. First of all, how widespread is systemic endocytosis? The induction of systemic endocytosis by flg22 supports the common existence of systemic endocytosis (Supplementary Fig. 8). REEs are also internalized in animal cells by CME, and this could provide a strategy for drug delivery in human ${ }^{17}$. It would be of great interest to know whether REEs could also induce systemic endocytosis in animals. Although seventeen REEs have similar physical and chemical properties, their biological effects are not completely identical ${ }^{4,14}$. It would be interesting to see if other REEs, such as $\mathrm{Ce}(\mathrm{III})$ or $\mathrm{Tb}(\mathrm{III})^{4,14}$, which also initiate endocytosis in leaf cells, could induce systemic endocytosis like $\mathrm{La}(\mathrm{III})$. In addition, it has been reported that direct application of REEs on roots could enhance endocytosis of root cells ${ }^{16,52}$. It would 
a
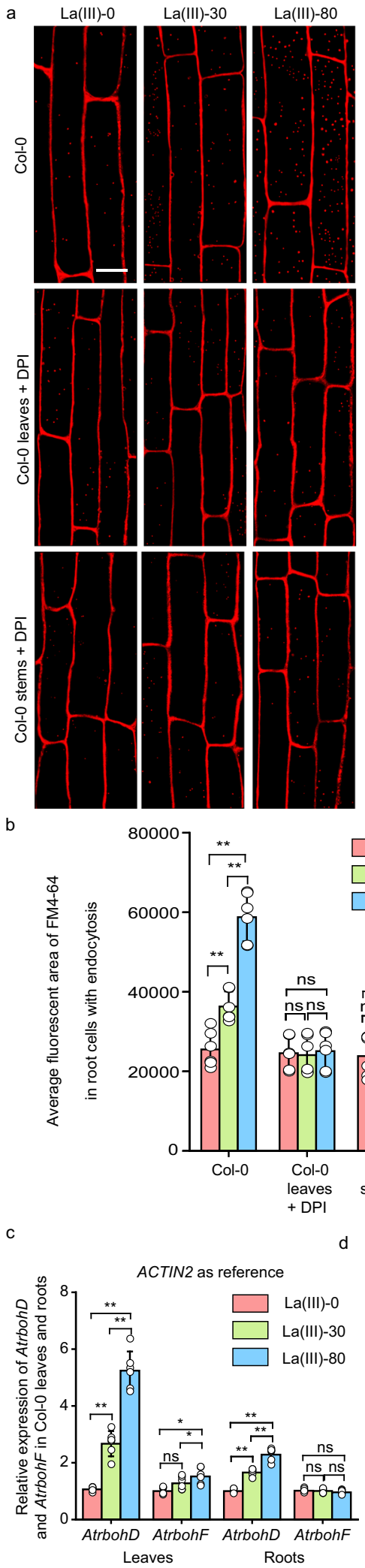

also be of importance to know whether the REE-induced systemic endocytosis is bidirectional, i.e., whether there is root-to-shoot systemic endocytosis as well.

Our findings show that the systemic endocytosis induced by La (III) is dependent on AtrbohD-mediated generation of a systemic signal (Figs. 1-5, Supplementary Figs. 1-10, and Supplementary Tables 1 and 2). Our data also suggest AtrbohD plays an

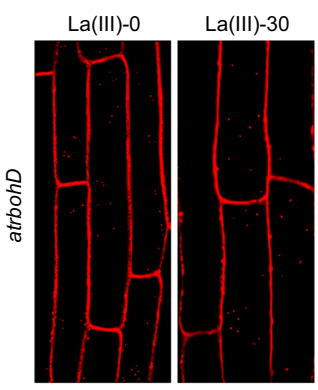

$\mathrm{La}(\mathrm{III})-80$
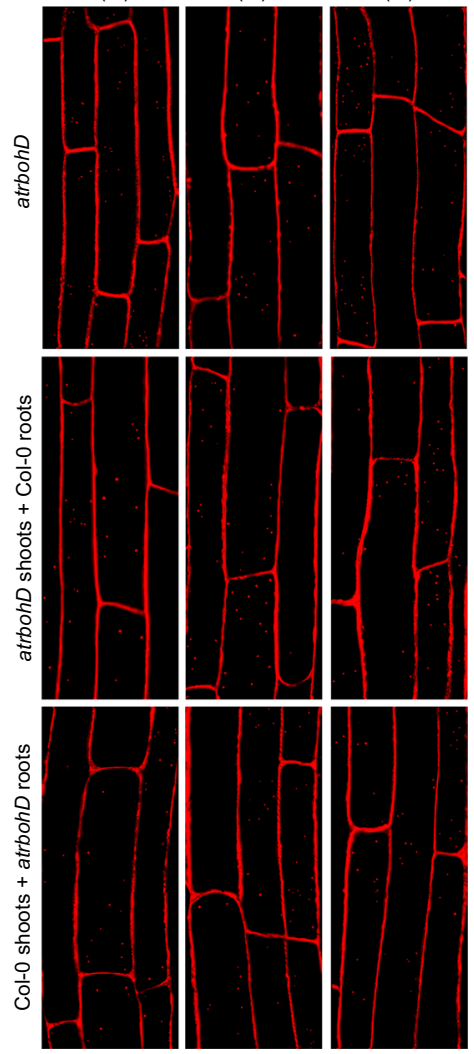

$\mathrm{La}(\mathrm{III})-0$

$\mathrm{La}(\mathrm{III})-30$

$\mathrm{La}(\mathrm{III})-80$
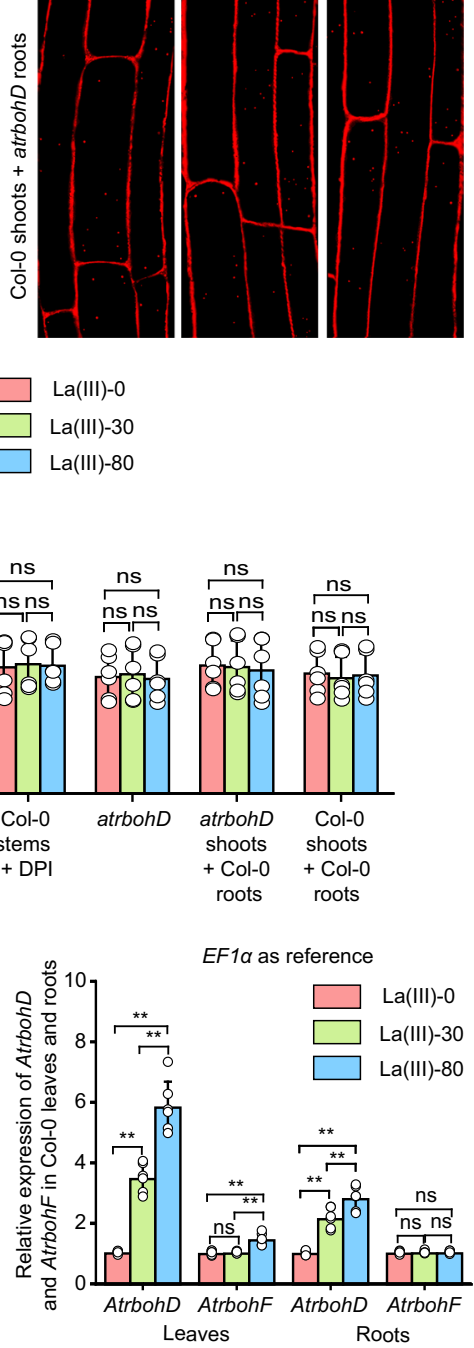

important role in the long-distance signal transmission by coordinating with another long-distance signal JA (Figs. 1-5, Supplementary Figs. 1-10, and Supplementary Tables 1-4). We found that AtrbohD regulates the accumulation of JA, and JA is further required for the production of the systemic endocytosis signals induced by $\mathrm{La}(\mathrm{III})$. Therefore, these findings support a paradigm for long-distance signal transmission, in which 
Fig. 3 Systemic endocytosis induced by La(III) was dependent on AtrbohD. a The representative CLSM images of Col-0, DPI-treated Col-0 (leaves or stems treated with $\mathrm{DPI}$ ), atrbohD, and grafted atrbohD (shoots from atrbohD were grafted onto Col-0 roots and shoots from Col-0 were grafted onto atrbohD roots) root cells. Leaves of all of these Arabidopsis were treated with 0, 30, or $80 \mu \mathrm{M} \mathrm{La(III)} \mathrm{for} 12 \mathrm{~h}$ and then roots were stained with FM4-64. $B a r=10 \mu \mathrm{m}$. Representative images from six independent measurements and three biological replicates (each replicate represents an independently treated plant) per measurement are represented. b Quantitative analysis of the average fluorescent area of FM4-64 in Col-0, Col-0 leaves or stems treated with DPI, atrbohD, and grafted atrbohD (shoots from atrbohD were grafted onto Col-0 roots and shoots from Col-0 were grafted onto atrbohD roots) root cells with endocytosis (data come from a). Values shown are means \pm SEM, one-way ANOVA analysis with LSD multiple comparisons test $\left(n=6\right.$, ${ }^{\star \star} p<$ 0.01). c, $\mathbf{d}$ The expression levels of AtrbohD and AtrbohF transcripts in Col-0 leaves and roots $12 \mathrm{~h}$ after treatment of leaves with 0,30 , or $80 \mu \mathrm{M}$ La(III). The expression levels were quantified by using quantitative RT-PCR. ACTIN2 (c) and EF1 $\alpha$ (d) were as two references. Six independent measurements and three replicates per measurement were conducted. Values shown are means \pm SEM, one-way ANOVA analysis with LSD multiple comparisons test $(n=6$, ${ }^{\star} p<0.05,{ }^{\star \star} p<0.01$, n.s.: no significance).
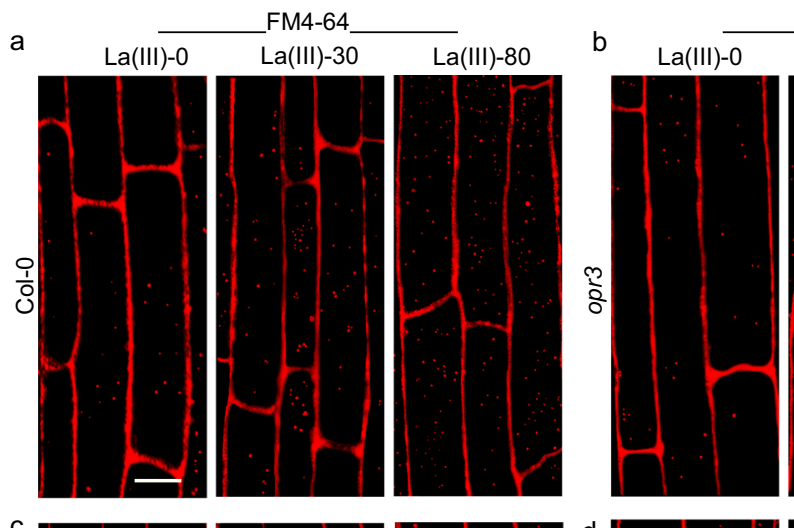

FM4-64
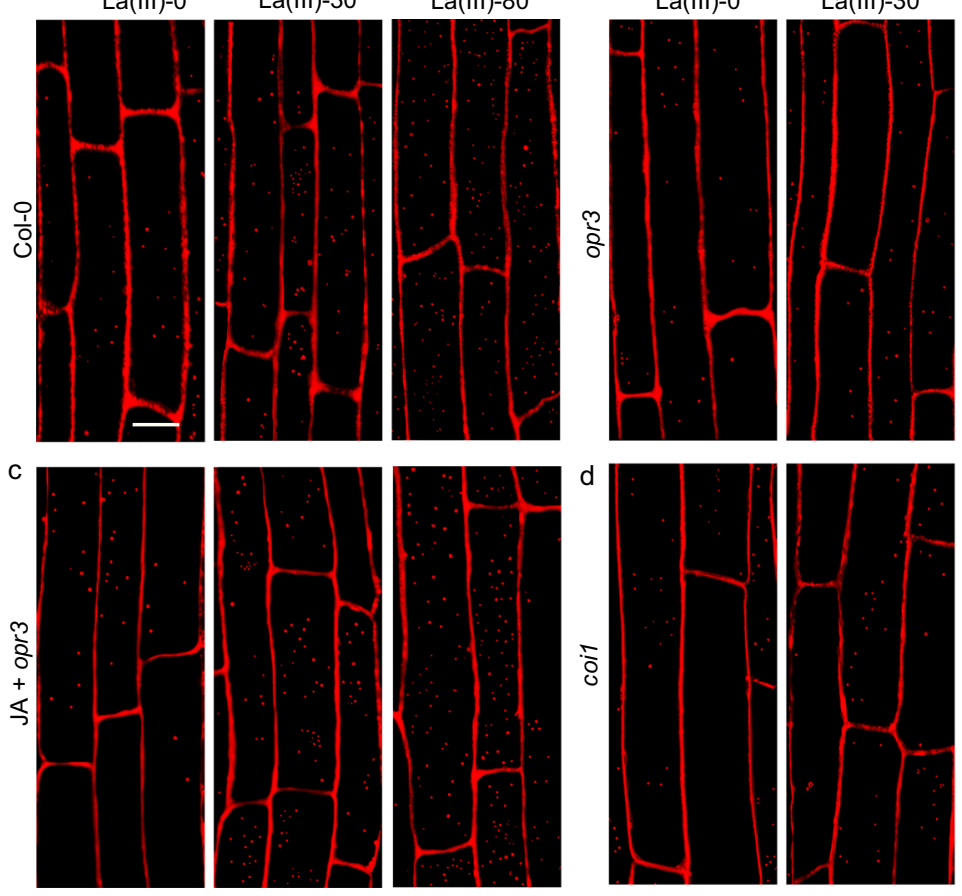

$\mathrm{La}(\mathrm{III})-80$

e

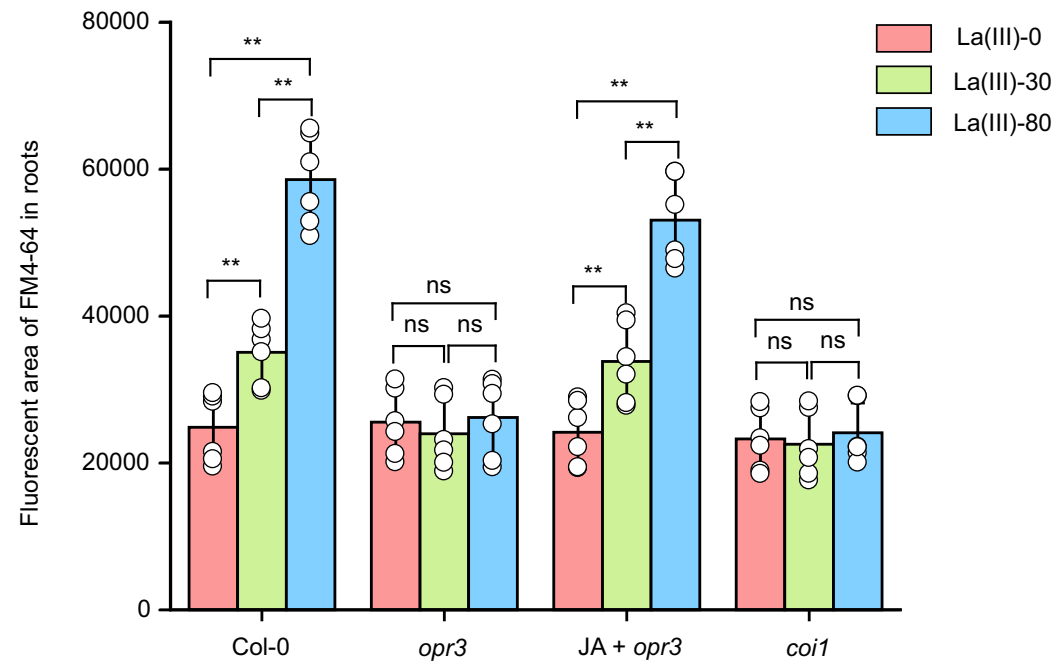

Fig. 4 The production of JA changed endocytosis. a-d Visualization of endocytosis labeled with FM4-64 in Col-0 (a), opr3 (b), JA-treated opr3 (c), and coil (d) roots $12 \mathrm{~h}$ after treatment of leaves with 0,30 , or $80 \mu \mathrm{M} \mathrm{La}(\mathrm{III})$. Bars $=10 \mu \mathrm{m}$. Representative images from six independent measurements and three biological replicates (each replicate represents an independently treated plant) per measurement are represented. e Quantitative analysis of the fluorescent area of FM4-64 in root cells of Col-0, opr3, JA-treated opr3, and coil (data come from a-d). Values shown are means \pm SEM. One-way ANOVA analysis with LSD multiple comparisons test $\left(n=6,{ }^{\star \star} p<0.01\right.$, n.s.: no significance). 


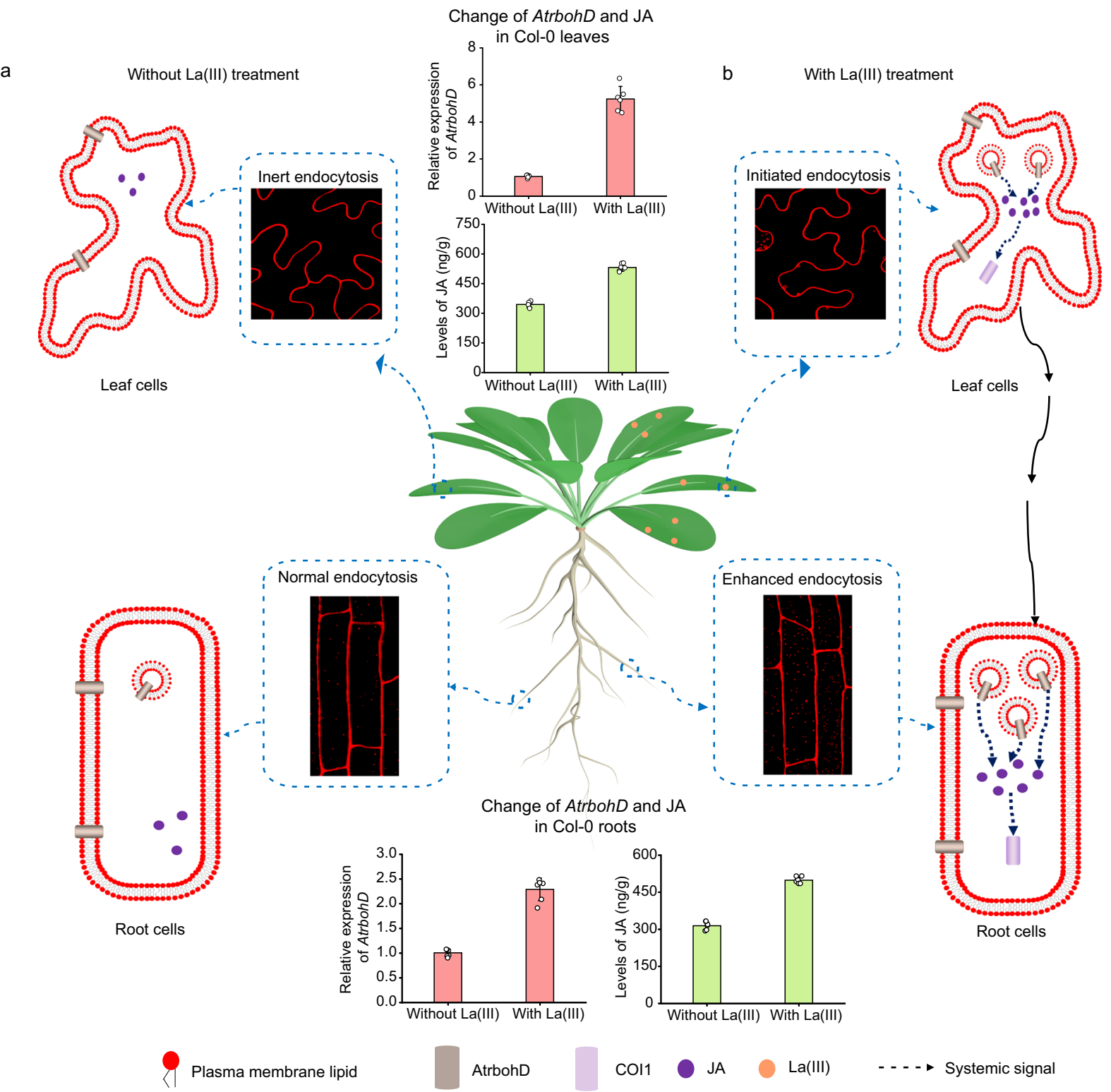

Fig. 5 A model for AtrbohD- and JA-dependent systemic endocytosis induced by La(III). a Without La(III) treatment, inert endocytosis and normal endocytosis were maintained in leaf and root cells, respectively. Meanwhile, small amounts of JA were produced in leaf and root cells. $\mathbf{b}$ La(III) treatment on leaves induced CME in leaf cells. AtrbohD promoted the accumulation of JA, and JA further transmitted systemic endocytic signal with the participation of COI1-dependent signaling. This systemic signal induced the enhanced CME in root cells.

AtrbohD-dependent signal and JA may self-propagate systemic endocytosis signaling over a long distance. How AtrbohD and JA regulate each other for the long-distance transmission of endocytosis signal and how the specificity of systemic signaling is determined will be important future questions. Under stress conditions, ROS is produced via NADPH oxidases and is proposed to propagate between cells as waves to transmit systemic signals over a long distance, which depends on hydrogen peroxide $\left(\mathrm{H}_{2} \mathrm{O}_{2}\right)$ in $\mathrm{ROS}^{39-43}$. It will be interesting to investigate whether $\mathrm{H}_{2} \mathrm{O}_{2}$ or other NADPH oxidase-dependent ROS coordinates with $\mathrm{JA}$ in the long-distance signaling that regulates systemic endocytosis.

REEs have been used in agriculture to improve crop productivity for decades, but the underlying mechanisms for REEs' regulation of plant growth remain unclear ${ }^{4,13-15,52-55}$. Interestingly our results suggest that $\mathrm{La}(\mathrm{III})$-induced systemic endocytosis regulates both leaf expansion and root development
(Supplementary Table 4). Furthermore, our findings show that La (III)-induced systemic endocytosis increases the accumulation of mineral nutrient elements in roots (Supplementary Table 3). Therefore, the REE-induced systemic endocytosis likely provides an explanation for REEs' effect on crop productivity. Nonetheless, elucidating the mechanisms by which the induced systemic endocytosis regulates the accumulation of mineral nutrient elements and plant growth will be an exciting and important future direction.

\section{Methods}

Reagents. $\mathrm{LaCl}_{3}$ (purity $>99.99 \%$ ) was purchased from Aladdin Bio-Chem Technology Co. Ltd (Shanghai, China). FM4-64 was purchased from Thermo Fisher Scientific Co. Ltd (Shanghai, China). DPI, TyrA23, Murashige and Skoog medium (MS), methyl 1-(butylcarbamoyl)-2-benzimidazolecarbamate (Benomyl), 6-benzylaminopurine, indole acetic acid (IAA), and JA were purchased from Sigma-Aldrich Co. Ltd (Shanghai, China). Flg22 was purchased from MedChemExpress Co. Ltd (Shanghai, China). Anti-plant actin mouse monoclonal antibody 
(A01050) was purchased from Abbkine Scientific Co. Ltd (Wuhan, China). Goat anti-mouse IgG H\&L [horseradish peroxidase (HRP)] (ab205719) and HRP antiGFP antibody (ab6663) were purchased from Abcam Co. Ltd (Shanghai, China). Other chemicals used in this study were analytical reagents and were purchased from Sinopharm Chemical Reagent Co. Ltd. (Shanghai, China).

Plant materials, growth conditions, and $\mathrm{LaCl}_{\mathbf{3}}$ treatment. Arabidopsis thaliana ecotypes Col-0, pCLC1::CLC1-GFP line ${ }^{28}$, DEX-inducible Venus-CANTH line ${ }^{15,35}$, and $a p 2 \mu$-1 (SALK_083693C) $)^{15}$, chc1-3 (SALK_018351C) $)^{34}$, atrbohD ${ }^{46}$, opr $3^{48}$, and coil $1^{50}$ mutants were used in this study. To generate a $p C L C 1$ ::CLC1-GFP line, the CLC1 genomic DNA including its upstream 271 bp was amplified by PCR. The PCR product was cloned into PDONOR to obtain an entry vector. Then, the entry vector was fused into the destination vector pGWB4 vector to obtain $p C L C 1::$ CLC1-GFP, which was transformed into A. thaliana Col-0 by the floral dipping method ${ }^{56}$. A recombinant plasmid for DEX-inducible expression of Venus-CANTH was constructed in a derivative of pTA7002, containing gateway cassettes kindly provided by Yuichiro Watanabe from the University of Tokyo ${ }^{57}$. A partial complementary DNA (cDNA) for the AP180 protein (residues 991-1959 of At1g05020) was amplified by PCR. The PCR product was fused into PDONOR to obtain an entry vector, which was fused into the derivative of pTA7002. The resulting plasmid was used to transform A. thaliana Col-0 to obtain a DEX-inducible VenusCANTH line. For induction, $5 \mu \mathrm{M}$ DEX from $30 \mathrm{mM}$ stock solution in ethanol was used. The primers used in generating CLC1-GFP and DEX-inducible VenusCANTH lines were listed in Supplementary Table 5.

Seeds were surface sterilized and imbibed for 2 days at $4{ }^{\circ} \mathrm{C}$ in the dark, and then plated onto an MS medium ( $\mathrm{pH}$ 5.7) supplemented with $1 \%(\mathrm{w} / \mathrm{v})$ sucrose and $1 \%$ agar $(\mathrm{w} / \mathrm{v})$ at $22^{\circ} \mathrm{C}$. Seedlings were grown in a climate-controlled growth room $\left[22^{\circ} \mathrm{C} / 20^{\circ} \mathrm{C}\right.$ (day/night), $100 \mu \mathrm{mol} \mathrm{m}{ }^{-2} \mathrm{~s}^{-1}$ light intensity and $16 / 8$-h photoperiod (day/night)]. Some five-day-old seedlings with healthy roots were transplanted into the soil and grown in the growth chamber for another 15 days.

All plant treatments, unless specified, were performed as follows: (1) 20-day-old seedlings were sprayed evenly with $5,30,55,80$, or $105 \mu \mathrm{M} \mathrm{LaCl}_{3}$ until droplets began to fall and the control seedlings were sprayed evenly with deionized water. Twelve hours, $24 \mathrm{~h}$, or 7 days after spraying, leaves and roots in the same position were sampled for further analyses. (2) Twenty-day-old seedlings were sprayed evenly with 90 or $240 \mu \mathrm{M} \mathrm{NaCl}$ until droplets began to fall and the control seedlings were sprayed evenly with deionized water. Twelve hours later, leaves and roots in the same position were sampled for further analyses. (3) Twenty-day-old seedlings were sprayed evenly with $10 \mu \mathrm{M}$ DPI or were treated with $10 \mu \mathrm{M}$ DPI in stems. Then, leaves were sprayed evenly with $\mathrm{LaCl}_{3}$ solution $(0,30$, or $80 \mu \mathrm{M})$ until the droplets began to fall. Twelve hours, $24 \mathrm{~h}$, or 7 days after spraying, leaves and roots in the same position were sampled for further analyses. (4) Twenty-day-old seedlings were sprayed evenly with $30 \mu \mathrm{M}$ TyrA23 $3^{25}$. One hour later, leaves were sprayed evenly with $\mathrm{LaCl}_{3}$ solution $(0,30$, or $80 \mu \mathrm{M})$ until droplets began to fall. Twelve hours, $24 \mathrm{~h}$, or 7 days after spraying, leaves and roots in the same position were sampled for further analyses. (5) Twenty-day-old seedlings were sprayed evenly with 0 or $10 \mu \mathrm{M}$ flg22 until droplets began to fall. Two hours later, leaves and roots in the same position were sampled for further analysis. (6) Twenty-dayold opr 3 was sprayed evenly with $50 \mathrm{nM} \mathrm{JA}$. Sixty minutes later, leaves were sprayed evenly with $\mathrm{LaCl}_{3}$ solution $(0,30$, or $80 \mu \mathrm{M})$ until the droplets began to fall. Twelve hours after spraying, leaves and roots in the same position were sampled for further analyses. (7) Roots of 20-day-old seedlings were treated with 0 or $50 \mathrm{nM} \mathrm{JA}$. Twelve hours after treatment, roots in the same position were sampled for further analyses.

Grafting of Arabidopsis plants. Seedlings to be grafted were germinated on plates containing $0.5 \times$ MS with $3 \mathrm{mg} \mathrm{L}^{-1}$ Benomyl, $0.04 \mathrm{mg} \mathrm{L}^{-1}$ 6-benzylaminopurine, $0.02 \mathrm{mg} \mathrm{L}^{-1} \mathrm{IAA}$, and $12 \mathrm{~g} \mathrm{~L}^{-1}$ agar. Treatment with IAA could be found to greatly improve grafting efficiency based on the following factors: enhanced callusing at the grafted union, retardation of shoot growth that maintained contact at the grafted union, and an $\sim 90 \%$ reduction in the formation of adventitious roots at the grafted union. Benomyl virtually eliminated fungal contamination. Plates containing the stratified seeds were placed vertically under controlled environmental conditions $\left(16 / 8\right.$-h photoperiod and $\left.25^{\circ} \mathrm{C}\right)$. Five-day-old seedlings were grafted on the plate by the $90^{\circ}$ blunt-end technique with a $15^{\circ}$ Stab Knife without collars ${ }^{58}$. The grafted seedlings remained on the plate for the next 5 days to allow the formation of the grafted union. Successfully unified seedlings were transplanted directly to the soil (as described above in the "Plant materials, growth conditions, and $\mathrm{LaCl}_{3}$ treatment" section).

\section{Measurements of growth, physiological, and biochemical indices. Growth} indices, including the total leaf areas of all leaves, primary root length, and lateral root numbers, were measured. Three independent measurements and ten plants per measurement were conducted. Physiological and biochemical indices, including net photosynthetic rate, chlorophyll content, and thiobarbituric acid-reactive species (TBARS) content, were measured as follows.

The net photosynthetic rate was measured using a portable fluorescence system (GFS-3000, WALZ, Effeltrich, Germany) in a chamber. Light saturation point, temperature, $\mathrm{CO}_{2}$ concentration, air flow rate, and relative humidity in the chamber were $800 \mu \mathrm{mol} \mathrm{m}^{-2} \mathrm{~s}^{-1}, 25^{\circ} \mathrm{C}, 700 \mathrm{mg} \mathrm{m}^{-3}, 750 \mu \mathrm{mol} \mathrm{s}^{-1}$, and $70 \%$, respectively.

Fresh leaves $(\sim 0.1 \mathrm{~g})$ were homogenized with $10 \mathrm{~mL}$ extraction solution $(80 \%$ acetone). Then, samples were centrifuged at $10,000 \times g$ for 5 min. The supernatant was collected and its absorbance was measured at 663 and $645 \mathrm{~nm}\left(A_{663}\right.$ and $\left.A_{645}\right)$ using an ultraviolet-visible spectrophotometer (Evolution ${ }^{\mathrm{TM}}$ 60S, Thermo Fisher Scientific, Shanghai, China), respectively. Chlorophyll content was calculated according to Arnon's equation as shown in the following equation ${ }^{59}$ :

$$
\text { Chlorophyll content }\left(\mathrm{mg} \cdot \mathrm{g}^{-1} \mathrm{FW}\right)=\frac{\left(8.02 A_{663}+20.21 A_{645}\right) \times V}{\mathrm{FW} \times 1000}
$$

In this equation, $V$ and $\mathrm{FW}$ were the volume of the extraction solution $(\mathrm{mL})$ and the fresh weight of leaves $(\mathrm{g})$, respectively.

Fresh leaves $(\sim 0.1 \mathrm{~g})$ were homogenized with $1 \mathrm{~mL}$ extraction solution $(10 \%$ trichloroacetic acid). Then, samples were centrifuged at $10,000 \times g$ for $10 \mathrm{~min}$. The supernatant was collected and $0.2 \mathrm{~mL} 0.6 \%$ thiobarbituric acid was added to $0.2 \mathrm{~mL}$ supernatant to obtain the reaction solution. After reacting in boiling water for 30 $\mathrm{min}$, the reaction solution was cooled and centrifuged at $10,000 \times g$ for $10 \mathrm{~min}$. The supernatant was collected and its absorbance was measured at 450,532 , and 600 $\mathrm{nm}\left(A_{450}, A_{532}\right.$, and $\left.A_{600}\right)$ using the ultraviolet-visible spectrophotometer, respectively. TBARS content was calculated according to the following equation:

TBARS content $\left(\mathrm{nmol} \cdot \mathrm{g}^{-1} \mathrm{FW}\right)=\frac{\left[6.45 \times\left(A_{532}-A_{600}\right)-0.56 \times A_{450}\right] \times V_{\mathrm{r}}}{\mathrm{FW} \times V_{\mathrm{s}} \div V_{\mathrm{e}}}$

In this equation, $\mathrm{FW}, V_{\mathrm{r}}, V_{\mathrm{s}}$, and $V_{\mathrm{e}}$ were the fresh weight of leaves $(\mathrm{g})$ and the volume of the reaction solution, supernatant in the reaction solution and the extraction solution $(\mathrm{mL})$, respectively.

Endocytosis observation. Leaves and roots were immersed in $2 \mu \mathrm{M}$ FM4-64 for $30 \mathrm{~min}$ and observed under a CLSM (Leica SP8, Wetzlar, Germany) with a $\times 63$ oil objective. The excitation wavelength for FM4-64 and CLC1-GFP was 514 and 488 $\mathrm{nm}$, respectively. The observation wavelength for FM4-64 and CLC1-GFP was 650 and $515 \mathrm{~nm}$, respectively. Moreover, the dynamic process of endocytosis was observed using spinning disc confocal microscopy with a CSU-X1 spinning disc head (Yokogawa, Tokyo, Japan) equipped with a CFI Apo TIRF 1003 NA1.49 oil immersion objective and an Evolve EMCCD camera.

For observing the morphology of endocytosis, leaves $(2-3 \mathrm{~mm}$ in length and width) were fixed in the solution containing $2 \%$ paraformaldehyde and $1 \%$ glutaraldehyde solution and postfixed with $1 \%$ osmic acid. After fixation and dehydration, samples were embedded in epoxy resin. Following epoxy resin polymerization, thin sections were prepared with a Reichert-Jung Ultracut $\mathrm{E}$ ultramicrotome (Vienna, Austria). Then, the thin sections were observed under a TEM (HITACH H-7650, Tokyo, Japan).

Western blotting assay. To investigate Venus-CANTH expression in the DEX inducible Venus-CANTH line, western blotting assay was performed. Total proteins were extracted from leaves by using $1 \mathrm{~mL}$ of Protein Extraction Buffer $[50 \mathrm{mM}$ Tris- $\mathrm{HCl}$ (pH 7.4), $5 \mathrm{mM}$ EDTA, $150 \mathrm{mM} \mathrm{NaCl}, 1 \%$ Triton X-100], $10 \mu \mathrm{L}$ of $100 \times$ phenylmethanesulfonyl fluoride, and $10 \mu \mathrm{L}$ of $100 \times$ protease inhibitor cocktail. Then, the samples were subjected to sodium dodecyl sulfate-polyacrylamide gel electrophoresis and analyzed by western blotting. Anti-plant actin mouse monoclonal antibody (1:2000 dilution) and monoclonal goat anti-mouse IgG H\&L (HRP) (1:2000 dilution) were used for detecting actin. HRP anti-GFP antibody (1:2000 dilution) was used for detecting Venus-CANTH.

Quantitative real-time PCR (qRT-PCR) analysis. To investigate the expression levels of AtrbohD, AtrbohF, and OPR3, qRT-PCR analysis was performed. Total RNA was isolated using an RNA purification kit. The total RNA was reverse transcribed using the PrimeScript RT Reagent Kit with gDNA Eraser. For qRTPCR analysis, the primers of AtrbohD, AtrbohF, and OPR3 were used to determine their expression levels, and these primers were listed in Supplementary Table 5. $A C T I N 2$ and EF1 $\alpha$ were used as references.

Osmotic pressure analysis. Fresh leaves or roots $(0.05 \mathrm{~g})$ were frozen with liquid nitrogen and squeezed in a syringe as soon as the frozen leaves melt. The liquid obtained from squeeze was centrifuged at $11,300 \times g$ for $5 \mathrm{~min}$. Then, the supernatant was diluted with deionized water and centrifuged to remove air bubbles. Osmotic pressure was measured using a freezing point osmometer (OSMOMAT 030, Berlin, Germany).

JA quantification. Fresh leaves or roots $(0.2 \mathrm{~g})$ were homogenized in an ice bath with $0.5 \mathrm{~mL}$ extraction buffer [isopropanol:water: $\mathrm{HCl}=2: 1: 0.005(\mathrm{v} / \mathrm{v} / \mathrm{v})]$ and agitated for $30 \mathrm{~min}$ at $4{ }^{\circ} \mathrm{C}$. Then, samples were dissolved in $200 \mu \mathrm{L}$ of $80 \%$ methanol after extraction with dichloromethane and solvent evaporation, and analyzed using LC (Agilent 1290 Infinity LC, Santa Clara, USA)-MS (Agilent 6460 QQQ MS, Santa Clara, USA). JA was separated on an Eclipse XD8-C18 HPLC column $\left(3 \mu \mathrm{m}, 150 \times 2.00 \mathrm{~mm}^{2}\right.$, Agilent, Santa Clara, USA) using a gradient of $0.1 \%$ formic acid in water (solvent $\mathrm{A}$ ) and $0.1 \%$ formic acid in acetonitrile (solvent B) at flow rate of $600 \mu \mathrm{L} \mathrm{min}{ }^{-1}$. 
Metal element and P level analysis. Twenty-four hours after spraying Col-0 seedlings with 0,30 , or $80 \mu \mathrm{M} \mathrm{LaCl}$, the roots were washed five times with deionized water and dried at $65^{\circ} \mathrm{C}$ until they were a constant weight. After grinding, the mixture was digested in concentrated nitric acid and $\mathrm{H}_{2} \mathrm{O}_{2}$ in a multiwave sample preparation system (Multiwave 3000, Anton Paar, Austria), in which $5 \mathrm{~mL}$ nitric acid and $0.5 \mathrm{~mL} \mathrm{H}_{2} \mathrm{O}_{2}$ were added in a Teflon perfluoroalkoxy digestion vessel. After $1 \mathrm{~h}$ digestion, the digestion solution was diluted with micro-filtered deionized water for five times. The supernatant was centrifuged at 4000 r.p.m. for $5 \mathrm{~min}$, and the levels of $\mathrm{La}$ and mineral nutrient elements $(\mathrm{K}, \mathrm{Ca}, \mathrm{Mg}, \mathrm{P}, \mathrm{Fe}$, and $\mathrm{Zn}$ ) were determined by XSERIES 2 ICP-MS (Thermo Fisher Scientific, Bremen, Germany). Moreover, the levels of mineral nutrient elements (K, $\mathrm{Ca}, \mathrm{Mg}, \mathrm{P}, \mathrm{Fe}$, and $\mathrm{Zn})$ in $\mathrm{La}(\mathrm{III})$ and TyrA23 co-treated Col-0, or La(III) and DPI co-treated Col-0 were also determined according to this method.

Statistical analyses. All experiments, unless specified, were conducted for six independent measurements (six times), and each treatment group in each measurement contained three replicates. The results are expressed as the mean \pm SEM. One-way analysis of variance with least significant difference multiple comparisons test $(p<0.05$ or $p<0.01)$ was used for the analysis of significant differences.

Reporting summary. Further information on research design is available in the Nature Research Reporting Summary linked to this article.

\section{Data availability}

All data included in the manuscript and/or Supplemental materials are available from the corresponding authors upon reasonable request. Source data are provided with this paper.

Received: 4 June 2018; Accepted: 11 June 2021;

Published online: 15 July 2021

\section{References}

1. Brown, P. H., Rathjen, A. H., Graham, R. D. \& Tribe, D. E. Rare earth elements in biological systems. Handb. Phys. Chem. Rare Earths 13, 423-452 (1990).

2. Hu, Z. Y., Richter, H., Sparovek, G. \& Schnug, E. Physiological and biochemical effects of rare earth elements on plants and their agricultural significance: a review. J. Plant Nutr. 27, 183-220 (2004).

3. Ahmad, H. R. et al. Nanomaterials in Plants, Algae, and Microorganisms (Academic Press, Cambridge, 2018).

4. Wang, L. H. et al. Rare earth elements activate endocytosis in plant cells. Proc. Natl Acad. Sci. USA 111, 12936-12941 (2014).

5. Adisa, I. O. et al. Role of cerium compounds in fusarium wilt suppression and growth enhancement in tomato (Solanum lycopersicum). J. Agric. Food Chem. 66, 5959-5970 (2018).

6. Cheisson, T. \& Schelter, E. J. Rare earth elements: Mendeleev's bane, modern marvels. Science 363, 489-493 (2019).

7. Errea, I. et al. Quantum crystal structure in the 250-kelvin superconducting lanthanum hydride. Nature 578, 66-69 (2020)

8. Huang, C. H. Rare Earth Coordination Chemistry: Fundamentals and Applications (Wiley, Hoboken, 2010).

9. Adeel, M. et al. Cryptic footprints of rare earth elements on natural resources and living organisms. Environ. Int. 127, 785-800 (2019).

10. Gulani, V. et al. Gadolinium deposition in the brain: summary of evidence and recommendations. Lancet Neurol. 16, 564-570 (2017).

11. Tur, C. et al. Assessing treatment outcomes in multiple sclerosis trials and in the clinical setting. Nat. Rev. Neurol. 14, 75-93 (2018)

12. Sweeney, M. D., Sagare, A. P. \& Zlokovic, B. V. Blood-brain barrier breakdown in Alzheimer disease and other neurodegenerative disorders. Nat. Rev. Neurol. 14, 133-150 (2018).

13. Chien, S. S. Peculiar effects of barium, strontium, and cerium on Spirogyra. Baranical Gaz. 63, 406-409 (1917).

14. Wang, L. H. et al. Abnormal pinocytosis and valence-variable behaviors of cerium suggested a cellular mechanism for plant yield reduction induced by environmental cerium. Environ. Pollut. 230, 902-910 (2017).

15. Wang, L. H. et al. Arabinogalactan protein-rare earth element complexes activate plant endocytosis. Proc. Natl Acad. Sci. USA 116, 14349-14357 (2019).

16. Liu, Y. Y., Wang, R. L., Zhang, P., Sun, L. L. \& Xu, J. Involvement of reactive oxygen species in lanthanum-induced inhibition of primary root growth. $J$. Exp. Bot. 67, 6149-6159 (2016).

17. Jin, W. H. et al. Lanthanide-integrated supramolecular polymeric nanoassembly with multiple regulation characteristics for multidrug-resistant cancer therapy. Biomaterials 129, 83-97 (2017).
18. Wang, P., Lombi, E., Zhao, F. J. \& Kopittke, P. M. Nanotechnology: a new opportunity in plant sciences. Trends Plant Sci. 21, 699-712 (2016)

19. Yang, X. P., Pan, H. P., Wang, P. \& Zhao, F. J. Particle-specific toxicity and bioavailability of cerium oxide $\left(\mathrm{CeO}_{2}\right)$ nanoparticles to Arabidopsis thaliana. J. Hazard. Mater. 322, 292-300 (2017).

20. Chuah, J. A. \& Numata, K. Stimulus-responsive peptide for effective delivery and release of DNA in plants. Biomacromolecules 19, 1154-1163 (2018)

21. Liu, W. S. et al. Spatially resolved localization of lanthanum and cerium in the rare earth element hyperaccumulator fern Dicranopteris linearis from China. Environ. Sci. Technol. 54, 2287-2294 (2020)

22. Cotruvo, J. A. The chemistry of lanthanides in biology: recent discoveries, emerging principles, and technological applications. ACS Cent. Sci. 5, 1496-1506 (2019)

23. Lee, E. et al. Rare earth elements induce cytoskeleton-dependent and PI4Passociated rearrangement of SYT1/SYT5 endoplasmic reticulum-plasma membrane contact site complexes in Arabidopsis. J. Exp. Bot. 71, 3986-3998 (2020).

24. Kitakura, S. et al. Clathrin mediates endocytosis and polar distribution of PIN auxin transporters in Arabidopsis. Plant Cell 23, 1920-1931 (2011).

25. Dhonukshe, P. et al. Clathrin-mediated constitutive endocytosis of PIN auxin efflux carriers in Arabidopsis. Curr. Biol. 17, 520-527 (2007).

26. Picco, A. \& Kaksonen, M. Quantitative imaging of clathrin-mediated endocytosis. Curr. Opin. Plant Biol. 53, 105-110 (2018).

27. Zhang, Y. et al. Change your Tplate, change your fate: plant CME and beyond. Trends Plant Sci. 20, 41-48 (2015).

28. $\mathrm{Li}, \mathrm{H}$. et al. The REN4 rheostat dynamically coordinates the apical and lateral domains of Arabidopsis pollen tubes. Nat. Commun. 9, 2573 (2018).

29. Paez Valencia, J., Goodman, K. \& Otegui, M. S. Endocytosis and endosomal trafficking in plants. Annu. Rev. Plant Biol. 67, 309-335 (2016).

30. Buchanan, B. B., Gruissem, W. \& Jones, R. L. Biochemistry and Molecular Biology of Plants (Wiley, Rockville, 2015).

31. Zhu, J. K. Abiotic stress signaling and responses in plants. Cell 167, 313-324 (2016).

32. Zwiewka, M., Nodzynski, T., Robert, S., Vanneste, S. \& Friml, J. Osmotic stress modulates the balance between exocytosis and clathrin-mediated endocytosis in Arabidopsis thaliana. Mol. Plant 8, 1175-1187 (2015).

33. Guo, X. S., Zhou, Q., Lu, T. H., Fang, M. \& Huang, X. H. Distribution and translocation of ${ }^{141} \mathrm{Ce}(\mathrm{III})$ in horseradish. Ann. Bot. 100, 1459-1465 (2007).

34. Larson, E. R., van Zelm, E., Roux, C., Marion-Poll, A. \& Blatta, M. R. Clathrin heavy chain subunits coordinate endo- and exocytic traffic and affect stomata movement. Plant Physiol. 175, 708-720 (2017).

35. Nagawa, S. et al. ROP GTPase-dependent actin microfilaments promote PIN polarization by localized inhibition of clathrin-dependent endocytosis. PLoS Biol. 10, e1001299 (2012).

36. Dejonghe, W. et al. Mitochondrial uncouplers inhibit clathrin-mediated endocytosis largely through cytoplasmic acidification. Nat. Commun. 7, 11710 (2016).

37. Robatzek, S., Chinchilla, D. \& Boller, T. Ligand-induced endocytosis of the pattern recognition receptor FLS2 in Arabidopsis. Gene. Dev. 20, 537-542 (2006).

38. Volk, A. P. D. \& Moreland, J. G. ROS-containing endosomal compartments: Implications for signaling. Method. Enzymol. 535, 201-224 (2014).

39. Mittler, R. et al. ROS signaling: The new wave? Trends Plant Sci. 16, 300-309 (2011).

40. Wrzaczek, M., Brosche, M. \& Kangasjarvi, J. ROS signaling loopsproduction, perception, regulation. Curr. Opin. Plant Biol. 16, 575-582 (2013).

41. Waszczak, C., Carmody, M. \& Kangasjarvi, J. Reactive oxygen species in plant signaling. Annu. Rev. Plant Biol. 69, 209-236 (2018).

42. Kollist, H. et al. Rapid responses to abiotic stress: priming the landscape for the signal transduction network. Trends Plant Sci. 24, 25-37 (2019).

43. Lew, T. T. S. et al. Real-time detection of wound-induced $\mathrm{H}_{2} \mathrm{O}_{2}$ signalling waves in plants with optical nanosensors. Nat. Plants 6, 404-415 (2020).

44. Miller, G. et al. The plant NADPH oxidase RBOHD mediates rapid systemic signaling in response to diverse stimuli. Sci. Signal. 2, ra45 (2009).

45. O’Donnell, B. V., Tew, D. G., Jones, O. T. \& England, P. J. Studies on the inhibitory mechanism of iodonium compounds with special reference to neutrophil NADPH oxidase. Biochem. J. 290, 41-49 (1993).

46. Torres, M. A., Dangl, J. L. \& Jones, J. D. G. Arabidopsis gp91phox homologues AtrbohD and AtrbohF are required for accumulation of reactive oxygen intermediates in the plant defense response. Proc. Natl Acad. Sci. USA 99 517-522 (2002)

47. Chico, J. M., Chini, A., Fonseca, S. \& Solano, R. JAZ repressors set the rhythm in jasmonate signaling. Curr. Opin. Plant Biol. 11, 486-494 (2008).

48. Chehab, E. W. et al. Intronic T-DNA insertion renders Arabidopsis opr3 a conditional jasmonic acid-producing mutant. Plant Physiol. 156, 770-778 (2011).

49. Weber, H. Fatty acid-derived signals in plants. Trends Plant Sci. 7, 217-224 (2002). 
50. Xie, D. X., Feys, B. F., James, S., Nieto-Rostro, M. \& Turner, J. G. COI1: an Arabidopsis gene required for jasmonate-regulated defense and fertility. Science 280, 1091-1094 (1998).

51. Sun, J. Q. et al. Jasmonate modulates endocytosis and plasma membrane accumulation of the Arabidopsis PIN2 protein. N. Phytol. 191, 360-375 (2011).

52. He, D., Xia, B. X., Zhou, Q., Wang, L. H. \& Huang, X. H. Rare earth elements regulate the endocytosis and DNA methylation in root cells of Arabidopsis thaliana. Chemosphere 227, 522-532 (2019).

53. Wang, L. H. et al. Responses of plant calmodulin to endocytosis induced by rare earth elements. Chemosphere 154, 408-415 (2016).

54. Yang, Q. et al. Vitronectin-like protein is a first line of defense against lanthanum (III) stress in Arabidopsis leaf cells. Environ. Exp. Bot. 130, 86-94 (2016).

55. Yang, Q., Wang, L. H., He, J. F., Yang, Z. B. \& Huang, X. H. Direct imaging of how lanthanides break the normal evolution of plants. J. Inorg. Biochem. 182, 158-169 (2018).

56. Zhang, X. R., Henriques, R., Lin, S. S., Niu, Q. W. \& Chua, N. H. Agrobacterium-mediated transformation of Arabidopsis thaliana using the floral dip method. Nat. Protoc. 1, 641-646 (2006).

57. Aoyama, T. \& Chua, N. H. A glucocorticoid-mediated transcriptional induction system in transgenic plants. Plant J. 11, 605-612 (1997).

58. Turnbull, C. G. N., Booker, J. P. \& Ottoline Leyser, H. M. Micrografting techniques for testing long-distance signalling in Arabidopsis. Plant J. 32, 255-262 (2002).

59. Arnon, D. I. Copper enzymes in isolated chloroplasts. Polyphenoloxidase in Beta vulgaris. Plant Physiol. 24, 1-15 (1949).

\section{Acknowledgements}

We thank J.M. Zhou (The University of British Columbia) for her help with fluorescence labeling test; C.F. Jiang (China Agricultural University) for providing atrbohD mutant; P. Liu (China Agricultural University) for providing opr3 and coil mutants. We also thank C.H. Huang (Peking University) and X.W. Deng (Peking University) for their valuable suggestions on the project. This work was supported by the Natural Science Foundation of China 21977051, 21371100, 21501068, 31170477, Ph.D. Programs Foundation of Ministry of Education of China 20130093120006, and the Priority Academic Program Development of Jiangsu Higher Education Institutions.

\section{Author contributions}

X.H. and Z.Y. designed research. M.C., L.W., Q.Z., D.H., and J.Z. performed research M.C., L.W., Q.Z., D.C., S.N., H.L., L.T., Z.G., X.H., and Z.Y. analyzed data. M.C., L.W., X.H., and Z.Y. wrote the paper.

\section{Competing interests}

The authors declare no competing interests.

\section{Additional information}

Supplementary information The online version contains supplementary material available at https://doi.org/10.1038/s41467-021-24379-z.

Correspondence and requests for materials should be addressed to X.H. or Z.Y.

Peer review information Nature Communications thanks Jiří Friml, Nobuhiro Suzuki, and the other, anonymous reviewer(s) for their contribution to the peer review of this work. Peer review reports are available.

Reprints and permission information is available at http://www.nature.com/reprints

Publisher's note Springer Nature remains neutral with regard to jurisdictional claims in published maps and institutional affiliations.

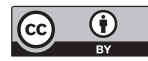

Open Access This article is licensed under a Creative Commons Attribution 4.0 International License, which permits use, sharing, adaptation, distribution and reproduction in any medium or format, as long as you give appropriate credit to the original author(s) and the source, provide a link to the Creative Commons license, and indicate if changes were made. The images or other third party material in this article are included in the article's Creative Commons license, unless indicated otherwise in a credit line to the material. If material is not included in the article's Creative Commons license and your intended use is not permitted by statutory regulation or exceeds the permitted use, you will need to obtain permission directly from the copyright holder. To view a copy of this license, visit http://creativecommons.org/ licenses/by/4.0/.

(C) The Author(s) 2021 